\title{
On the Design of Mobility Management Scheme for 802.16-based Network Environment
}

\author{
Junn-Yen $\mathrm{Hu}$ and Chun-Chuan Yang \\ Multimedia and Communications Laboratory \\ Department of Computer Science and Information Engineering \\ National Chi Nan University, Taiwan, R.O.C. \\ ccyang@csie.ncnu.edu.tw
}

\begin{abstract}
In this paper, we investigate the characteristics of IEEE 802.16 and conclude that it is better to equip BS and SS with Layer 3 functionality such that an 802.16 network acts as the backbone network of different subnets to enhance 802.16-based network deployment. Moreover, in order to support mobility in an 802.16 network environment, a novel concept called middle-domain mobility management for 802.16 is proposed in the paper. The 802.16 middle-domain is neither macro- nor micro-domain, but something in between them. By introducing the middle-domain in 802.16, an efficient mobility management scheme that can accommodate different micro-mobility protocols in an 802.16 network environment can be provided. Mobility management and handoff schemes as well as the associated cache structure for 802.16 middle-domain are presented in the paper.
\end{abstract}

Keywords: 802.16, BWA, Mobility Management

\section{INTRODUCTION}

Broadband Wireless Access (BWA) technology provides an easy, time-saving, and low-cost method for deployment of next generation (beyond 3G) network infrastructure. Since 1998, IEEE 802.16 working group has launched a standardization process called Wireless Metropolitan Area Network (Wireless MAN ${ }^{T M}$ ) for BWA. The newly released specification of 802.16 (IEEE Std 802.16-2004) [1] focuses on fixed location wireless access and can support up to $134 \mathrm{Mbps}$ bit rate. The WiMax Forum (Worldwide Interoperability for Microwave Access) [2, 3], a wireless industry consortium with about 100 members including such major vendors as $A T \& T$, Fujitsu, Intel, and Siemens Mobile, is supporting 802.16 technology and promoting its commercial use, which means 802.16 is becoming the most important technology in BWA.

As illustrated in Figure 1, a basic 802.16 network consists of a base station $(B S)$ and a couple of subscriber stations $(S S)$ that connects to the BS via high-speed wireless link. The BS acts as a gateway to the Internet. Legacy LANs or even more complex subnet systems can connect to the 802.16 network via SS. An 802.16 network (including the Legacy LANs that connects to SS) can cover a large geographical area since the distance between BS and SS can be up to 30 miles.

Similar to other 802 protocols, IEEE 802.16 defines the specification in physical layer (Layer 1) and MAC layer

This work was supported in part by the National Science Council, Taiwan, R.O.C., under grant NSC 93-2219-E-260-004

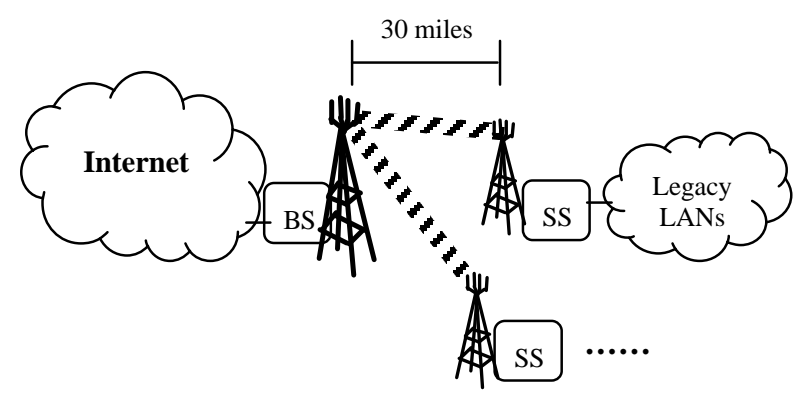

Figure1. General view of 802.16 network

(Layer 1.5). Thus, from the viewpoint of layering architecture in networking, an 802.16 network is basically a subnet and the BS or SS acts as a Layer 2 (L2) device (bridge, for instance). However, it is improper to view an 802.16 network as a subnet like 802.3 or 802.11 LAN, since (1) an 802.16 network can cover a large geographical area, and (2) a large number of users (including mobile hosts) in the network would cause serious performance degradation if the whole 802.16 network is only a single broadcast domain.

For example, Address Resolution Protocol (ARP) requires the ARP request frame to be broadcast in the whole 802.16 subnet in order to get the mapping from the logical IP address to the physical address. Moreover, in order to support mobile computing in 802.16, L2 mobility management as well as L2 handoff control [4] require the handoff frames to be broadcast in the network, creating more annoyed broadcast frames in the 802.16 network.

Therefore, we conclude that it is better to equip BS and SS with Layer 3 (L3) functionality such that 802.16 network acts as the backbone network of different subnets to enhance 802.16-based network deployment. This kind of network deployment (heterogeneous subnets interconnected by L3 $802.16 \mathrm{BS} / \mathrm{SS}$ ) is actually a form of internet, and it is called 802.16 network environment in this paper.

There are two approaches to support mobility in a 802.16 network environment: (1) 802.11 users roaming among WLANs or cellular systems that connects to SS, or (2) mobile hosts equipped with 802.16 interface connects to the BS directly. The standardization process for mechanisms supporting mobility via 802.16 interface (IEEE 802.16e, wireless access with high mobility) is still underway, so we 
focus on the case of approach (1) in this paper.

Given that 802.16 BS and SS are equipped with L3 functionality, an 802.16 network environment is beyond the ability of a Layer 2 mobility management scheme. Hence, we investigated the feasibility of applying existing L3 mobility management schemes in 802.16. We have found that the current two-tier mobility management (macro-mobility + micro-mobility) [5, 6] cannot fit in 802.16 network well. Therefore, a new concept of middle-domain mobility management for 802.16 network environment is proposed.

The rest of the paper is structured as follows. First of all, we make a brief survey of existing L3 mobility protocols in section II, and in section III, we discuss the problems of fitting existing mobility protocols in 802.16 . The concept of 802.16 middle-domain is presented in section IV. Simulation study is presented in section V. Finally, section VI concludes this paper.

\section{RELATED WORK}

Currently L3 mobility management solutions can be broadly classified into two categories: macro-mobility and micro-mobility management solutions, in which the movement of mobile users between two network domains is referred to as macro-mobility and the movement between two subnets within one domain is referred to as micro-mobility. In the following, we make a brief survey on the most typical macro-mobility protocol, Mobile IP $(M I P)$, and two typical micro-mobility protocols [7], Cellular IP (CIP) and Mobile IP Regional Registration (MIP-RR).

\section{A. Macro-mobility protocol: MIP}

In MIP [8, 9], a mobile host $(M H)$ uses two IP addresses: a fixed home address and a care-of-address (CoA) that changes at each new point of attachment (subnet). A router called Home Agent (HA) on an MH's home network is responsible for maintaining the mapping (binding) of the home address to the CoA. When an $\mathrm{MH}$ moves to a foreign network, the $\mathrm{MH}$ obtains a CoA from the Foreign Agent (FA) and registers the CoA with its HA. In this way, whenever an $\mathrm{MH}$ is not attached to its home network, the HA gets all packets destined for the $\mathrm{MH}$ and arranges to deliver to the MH's current point of attachment by tunneling the packets to the MH's CoA.

\section{B. Micro-mobility protocols: $C I P$ and MIP-RR}

CIP $[10,11]$ is proposed to provide local mobility and handoff support for frequently moving hosts. It supports fast handoff and paging in CIP access networks. For mobility between different CIP networks, it can interwork with MIP to provide wide-area mobility support. A Cellular IP network consists of a gateway (GW) and base stations (BS). The gateway connects the Cellular IP network to Internet. Cellular IP base stations are nodes that have an interface to a wireless network and interfaces to the wired network. Packets transmitted from mobile hosts are always routed from the base

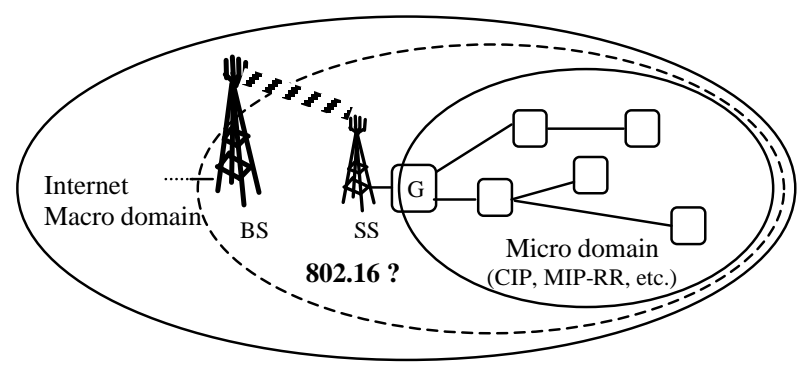

Figure 2. Position of 802.16 in mobility management

station to the gateway by a hop-by-hop shortest path routing. On the other hand, packets destined to an $\mathrm{MH}$ reach the GW first. Then the GW forwards the packets to the MH using the host-specific routing path.

MIP-RR [12] (or Hierarchical MIP, HMIP) aims to reduce the number of signaling messages to the home network and also reduce the signaling delay by performing registrations locally in a regional network. When an MH first arrives at a regional network, it performs a home registration with its HA. During the home registration, the HA registers the $\mathrm{CoA}$ of the $\mathrm{MH}$, which is actually a publicly routable address of another mobility agent called a gateway foreign agent (GFA). When an MH changes FAs within the same regional network, it performs only a regional registration to the GFA to update its local CoA. The packets for the MH are first intercepted by its HA, which tunnels those to the registered GFA. The GFA checks its visitor list and forwards the packets to the corresponding FA of the MH. The FA further relays the packets to the MH. In order to enhance the efficiency of mobility management in MIP-RR, more levels of Regional Foreign Agent (RFA) can be added between GFA and FA.

\section{Problems of Fitting Existing Protocols in 802.16}

Currently the two-tier mobility management uses the macro-mobility protocol and micro-mobility protocols at the same time but in different levels. The operation range of the macro-mobility protocol (MIP) is called the macro-domain and the operation range of a micro-mobility protocol such as CIP or MIP-RR is called a micro-domain in this paper.

Given that 802.16 devices are equipped with L3 functionality, and from the viewpoint of mobility management, 802.16 devices are used to connect different micro-domains to Internet, thus 802.16 is something between macro-domain and micro-domain as displayed in Figure 2. There are two straightforward ways to design mobility management in an 802.16 network environment: (1) macro-domain coupling, or (2) micro-domain coupling, as explained in the following.

\section{A. Macro-domain coupling}

As illustrated in Figure 3, we can simply treat 802.16 devices as part of the macro-domain. In such case, BS as well as SS of 802.16 can get rid of mobility management and act just like regular routers. Moreover, in addition to functions of 


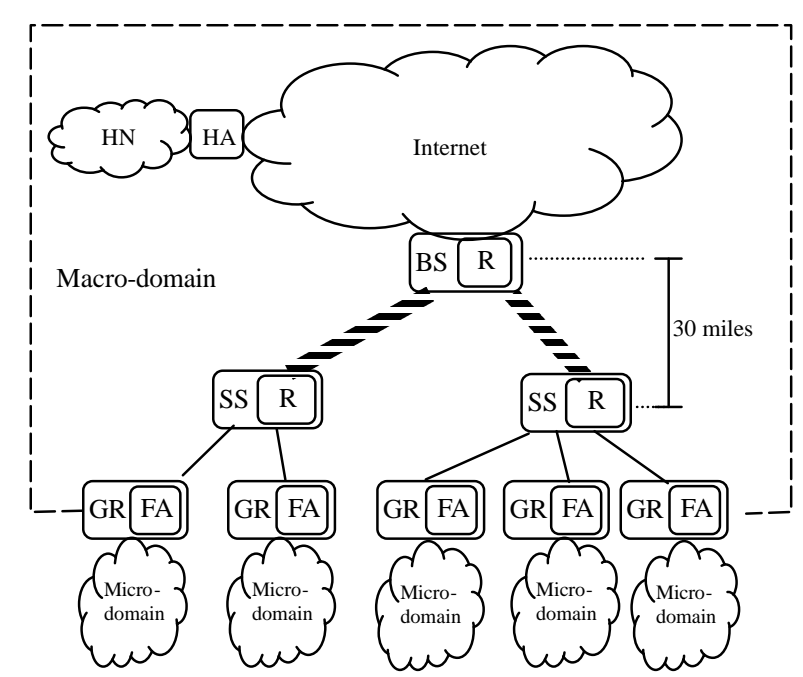

Figure 3. Coupling 802.16 with the macro-domain

micro-mobility protocol, the gateway router (GR) of each micro-domain is equipped with MIP FA functions and is responsible for MIP home registration. However, this kind of coupling introduces performance problem in mobility management, since MIP home registration is required for the handoffs between different micro-domains in the same 802.16 environment, which is inappropriate from the viewpoint of efficiency.

\section{B. Micro-domain coupling}

We can also treat the whole 802.16 network environment as a single micro-domain, in which CIP or MIP-RR can be applied to support mobility management. Figure 4 shows the typical examples for applying CIP and MIP-RR in 802.16 respectively. As illustrated in Figure 4-(a), since CIP requires all data packets to be routed to the gateway (BS of 802.16 in the case) before being routed to the destination, it results in a bad consequence that for internal traffic of which the source $\mathrm{MH}$ and the destination $\mathrm{MH}$ of the data packets are in the same 802.16 network, the traffic is routed to the BS first, even if the two mobile hosts are on neighboring subnets. The performance problem is called "lengthy internal data path", which also results in the waste of precious link bandwidth between BS and SS.

Figure 4-(b) shows the case of applying MIP-RR in an 802.16 network environment, in which each SS is equipped with the function of RFA to avoid the problem of lengthy internal data path. However, this case introduces another type of deployment problem called "one-hop tunneling" between BS and SS. Since the idea of tunneling in mobility support is used for packet transmission across networks (regular routers) that are not supporting mobility scheme, one-hop tunneling is inappropriate and inefficient.

Last but not least, since an 802.16 network environment can cover a large area, treating it as a single micro-domain lacks for the flexibility of adopting different mobility protocols in different micro-domains.

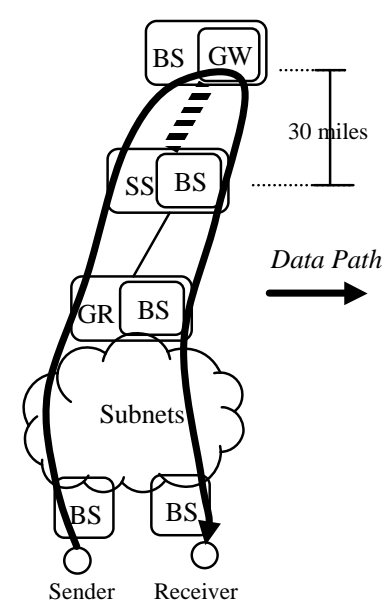

(a) Lengthy internal data path in CIP

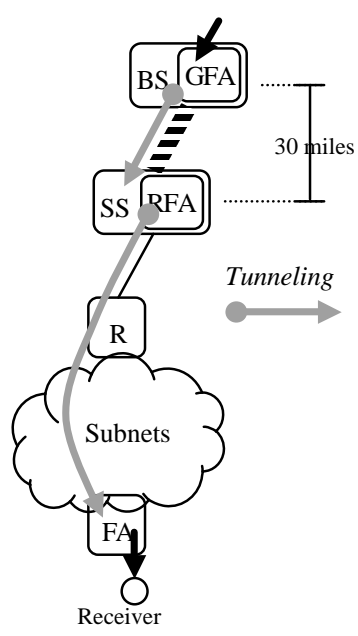

(b) One-hop tunneling in MIP-RR
Figure 4. Coupling 802.16 with a micro-domain

\section{802.16 Middle-Domain Mobility Management}

\section{A. Basic idea}

We conclude from the discussion in section III that it is not appropriate to treat an 802.16 network environment as part of the macro-domain nor a single micro-domain. Therefore, the idea of middle-domain emerges. Introducing the 802.16 middle-domain results in a 3-tier mobility management as illustrated in Figure 5. Given that the idea of middle-domain is created after the 2-tier mobility management, the operations of 802.16 middle-domain are designed to be transparent from the viewpoint of macro- or micro-domains. That is, neither MIP nor micro-mobility protocols is required to be aware of the existence of the middle-domain, and the operations of MIP as well as micro-mobility protocols remain the same.

\section{B. Location Management}

As in the 2-tier mobility management, the micro-domain gateway router (GR) under each SS is required to equipped with MIP FA functions and is responsible for MIP home registration. But the registration requests issued by the GR are intercepted by SS or BS in order to perform proper actions of the middle-domain. If an $\mathrm{MH}$ enters the middle-domain the first time, the following actions are taken in the middle-domain based on the intercepted MIP registration request:

(1) The BS and SS en route create the location cache for the corresponding $\mathrm{MH}$.

(2) The BS allocates a middle-domain $\operatorname{CoA}$ (denoted by $M-C o A$ in the paper) for the MH. The M-CoA is usually the address of the BS and is used in MIP registration.

(3) The BS issues an MIP registration request with the M-CoA to the MH's HA on behalf of the GR. Meanwhile, the BS sends an MIP reply message back to the GR on 


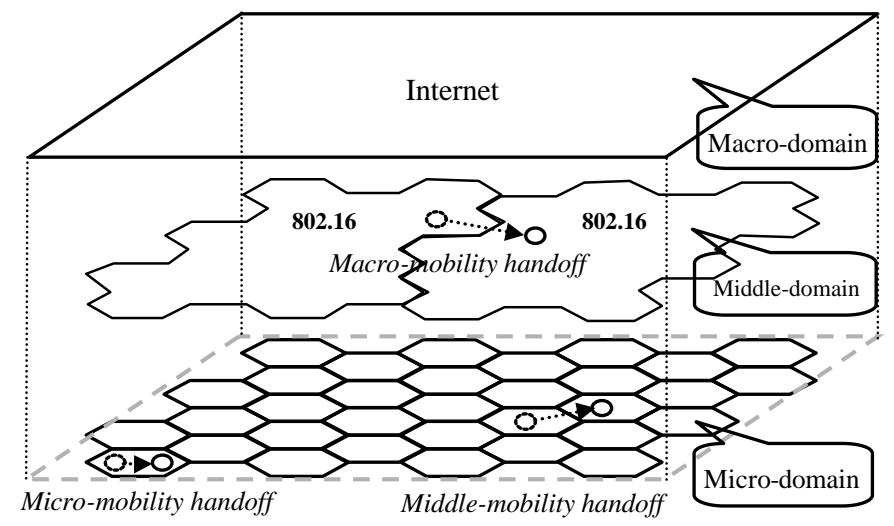

Figure 5. The idea of 802.16 middle-domain

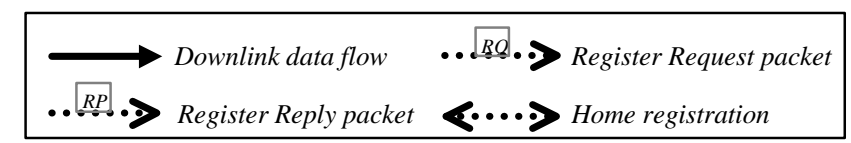

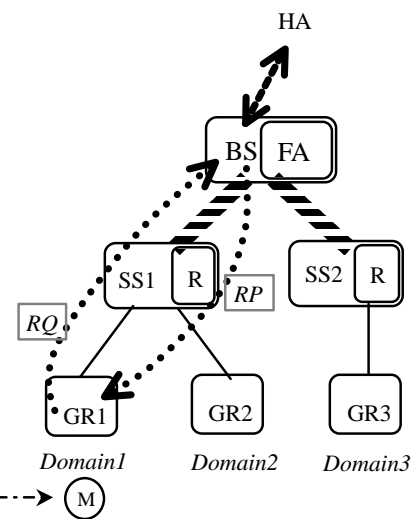

(a) Signaling flow

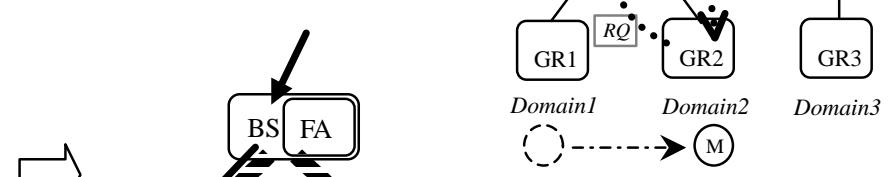

(a) Intra-SS handoff
BS: \begin{tabular}{|l|l|l|l|}
\hline MH's ID & Next Hop & M-CoA & Micro-domain CoA \\
\hline
\end{tabular}

SS: \begin{tabular}{|l|l|l|}
\hline MH's ID & Next Hop & Micro-domain CoA \\
\hline
\end{tabular}

Figure 7. Cache structure in the middle-domain

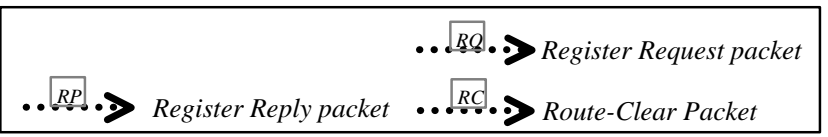

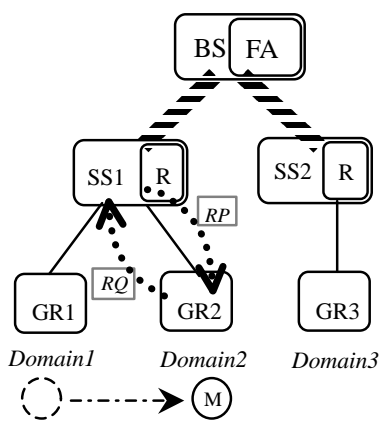

Figure 8. Handoff scheme in 802.16 middle-domain in the previous SS must be cleared. Therefore, a new control packet called route-clear packet is defined in the middle-domain. Signaling flow for inter-SS but intra-BS handoff is illustrated in Figure 8-(b). Note that there is no need to perform MIP home registration for both cases.

\section{Data Delivery}

Data delivery from the $\mathrm{CN}$ to an $\mathrm{MH}$ with the introduction of the middle-domain is explained as follows. As illustrated in Figure 9, data packets destined to an MH's home address are first intercepted by the HA. Since the CoA registered for the $\mathrm{MH}$ is the $\mathrm{M}-\mathrm{CoA}$, the HA tunnels the packets to the BS that allocated the M-CoA. The BS decapsulates the received packets and forwards them to the correct GR according to the location cache maintained by the BS and SS. Lastly, forwarding of the packets within a micro-domain is based on the operations of the micro-mobility protocol, which can be either tunneling-based (e.g. MIP-RR) or routing-based (e.g. CIP).

Similar to CIP, data packets transmitted by an $\mathrm{MH}$ in 802.16 are forwarded towards BS. However, the handling of the internal data flow is more efficient in the middle-domain as explained in the following. Since BS and SS maintain the location cache for each mobile host and if the data packets are destined to another $\mathrm{MH}$ in the same 802.16, the crossover BS/SS of the source micro-domain and the destination micro-domain will identify the corresponding location cache for the destination $\mathrm{MH}$ and relay the data packets to the correct next hop. 


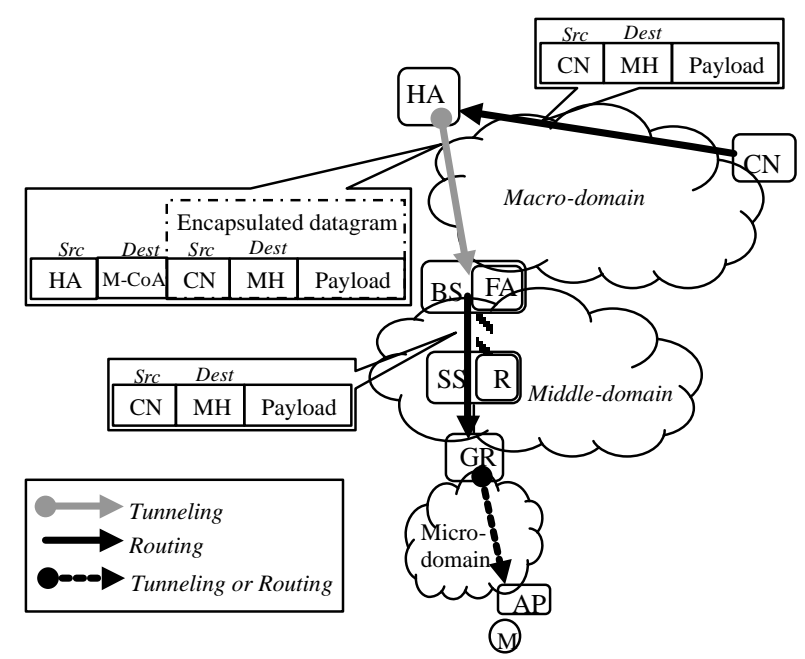

Figure 9. Data delivery in 3-tier mobility management

\section{Performance Evaluation}

Two 802.16 networks are created in the simulation. There is only one BS in each network. Four subscriber stations are connected to the BS, and four micro-domains are connected to each SS. The mobile hosts in the simulation leave the current micro-domain and handoff to one of the neighboring micro-domains with probability $20 \%$. The average signaling cost as well as the average handoff latency are displayed in Figure 10, which demonstrates the performance improvement of the middle-domain over the case without middle-domain.

\section{CONCLUSION}

Broadband Wireless Access (BWA) technology provides an easy, time-saving, and low-cost method for deployment of next generation (beyond $3 \mathrm{G}$ ) network infrastructure. The newly released specification of 802.16 (IEEE Std 802.16-2004) focuses on fixed location wireless access and can support up to $134 \mathrm{Mbps}$ bit rate. Since an 802.16 network can cover a large geographical area and support a large number of users, we conclude that it is better to equip BS and SS with Layer 3 functionality such that 802.16 network acts as the backbone network of different subnets to enhance 802.16-based network deployment.

Moreover, in order to support mobility in an 802.16 network environment, a novel concept called middle-domain mobility management for 802.16 is proposed in the paper. The 802.16 middle-domain is neither macro- nor micro-domain, but something in between them. By introducing the middle-domain in 802.16, an efficient mobility management scheme that can accommodate different micro-mobility protocols in an 802.16 network environment can be provided. Operations of BS and SS to support the middle-domain mobility management are designed to be transparent to the macro-domain as well as micro-domain protocols. Mobility management and handoff schemes as well as the associated

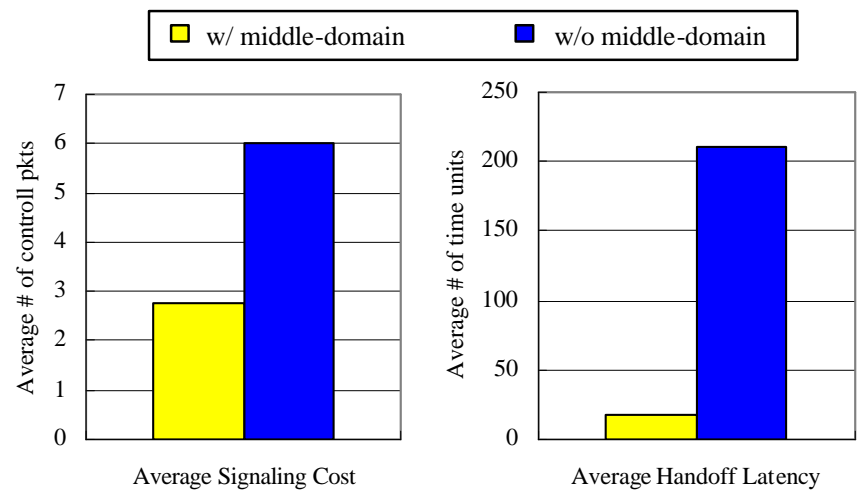

Figure 10. Simulation results

cache structure for 802.16 middle-domain are presented in the paper. Simulation study demonstrates the performance improvement of the middle-domain over the case without middle-domain in terms of less signaling cost and shorter handoff latency.

\section{REFERENCES}

[1] IEEE 802.16-2004, "IEEE Standard for Local and Metropolitan Area Networks-Part 16: Air Interface for Fixed Broadband Wireless Access Systems," 1 Oct. 2004.

[2] "Business Case Models for Fixed Broadband Wireless Access based on WiMAX Technology and the 802.16 Standard," WiMax Forum, 10 Oct. 2004.

[3] S. J. Vaughan-Nichols, "Achieving Wireless BroadBand with WiMax," Computer, pp.10-13, Jun. 2004.

[4] IEEE 802.11f/D5, "Draft Recommended Practice for Multi-Ventor Access Point Interoperability via an Inter-Access Point Protocol Across Distribution Systems Supportint IEEE 802.11 Operation.”Jan. 2003.

[5] M. Carli, A. Neri, and A. R. Picci, "Mobile IP and Cellular IP Integration for Inter Access Networks Handoff," Proc. IEEE International Conference on Communications (ICC), vol. 8, pp. 2467-2471, 2001.

[6] I. F. Akyildiz, J. Xie, and S. Mohanty, "A Survey of Mobility Management in Next-Generation All-IP-based Wireless Networks," IEEE Wireless Communications, pp. 16-28, Aug. 2004.

[7] A. T. Campbell, J. Gomez, S. Kim, and C.-Y. Wan, "Comparison of IP Micromobility Protocols," IEEE Wireless Communications, pp. 72-82, Feb. 2002.

[8] C. Perkins, "Mobile IP," IEEE Communication Magazine, vol. 35, issue 5, pp. 84-99, May 1997.

[9] C. Perkins, Ed., "IP Mobility Support for IPv4," IETF RFC 3344, August 2002.

[10] A.T. Campbell and J. Gomez, "An Overview of Cellular IP," Proc. IEEE Wireless Communications and Networking Conference (WCNC), pp. 606-610, 1999.

[11] A.T. Campbell, J. Gomez, S. Kim, A.G. Valko, C.Y. Wan, and Z.R. Turanyi, "Design, Implementation, and Evaluation of Cellular IP," IEEE Personal Communications, vol. 7, issue 4, pp. 42-49, Aug. 2000.

[12] C. Perkins, "Mobile IPv4 Regional Registration," Internet draft, draft-ietf-mobileip-reg-tunnel-09.txt, June 2004. 